This item was submitted to Loughborough's Research Repository by the author.

Items in Figshare are protected by copyright, with all rights reserved, unless otherwise indicated.

\title{
Robust hand-eye calibration of 2D laser sensors using a single-plane calibration artefact
}

PLEASE CITE THE PUBLISHED VERSION

https://doi.org/10.1016/j.rcim.2019.101823

\section{PUBLISHER}

(C) Elsevier

\section{VERSION}

AM (Accepted Manuscript)

\section{PUBLISHER STATEMENT}

This paper was accepted for publication in the journal Robotics and Computer-Integrated Manufacturing and the definitive published version is available at https://doi.org/10.1016/j.rcim.2019.101823.

\section{LICENCE}

CC BY-NC-ND 4.0

\section{REPOSITORY RECORD}

Sharifzadeh, Sara, Istvan Biro, and Peter Kinnell. 2019. "Robust Hand-eye Calibration of 2D Laser Sensors Using a Single-plane Calibration Artefact”. Loughborough University. https://hdl.handle.net/2134/38268. 


\title{
Robust hand-eye calibration of 2D laser sensors using a single-plane calibration artefact
}

\author{
Sara Sharifzadeh ${ }^{1}$, Istvan Biro ${ }^{2}$, Peter Kinnell ${ }^{2}$ \\ 1. School of Computing, Electronics and Maths, Faculty of Engineering, Environment \& \\ Computing, Coventry University, UK. \\ 2. EPSRC Centre for Innovative Manufacturing in Intelligent Automation Wolfson \\ School of Mechanical, Electrical and Manufacturing Engineering Loughborough \\ University, UK
}

\begin{abstract}
When a vision sensor is used in conjunction with a robot, hand-eye calibration is necessary to determine the accurate position of the sensor relative to the robot. This is necessary to allow data from the vision sensor to be defined in the robot's global coordinate system. For 2D laser line sensors hand-eye calibration is a challenging process because they only collect data in two dimensions. This leads to the use of complex calibration artefacts and requires multiple measurements be collected, using a range of robot positions. This paper presents a simple and robust hand-eye calibration strategy that requires minimal user interaction and makes use of a single planar calibration artefact. A significant benefit of the strategy is that it uses a low-cost, simple and easily manufactured artefact; however, the lower complexity can lead to lower variation in calibration data. In order to achieve a robust hand-eye calibration using this artefact the impact of robot positioning strategies is considered to maintain variation. A theoretical basis for the necessary sources of input variation is defined by a mathematical analysis of the system of equations for the calibration process. From this a novel strategy is specified to maximize data variation by using a circular array of target scan lines to define a full set of required robot positions. A simulation approach is used to further investigate and optimise the impact of robot position on the calibration process, and the resulting optimal robot positions are then experimentally validated for a real robot mounted laser line sensor. Using the proposed optimum method, a semi-automatic calibration process, which requires only four manually scanned lines, is defined and experimentally demonstrated.
\end{abstract}

\section{Keywords}

Hand-eye calibration, Laser line sensor, Robotic metrology.

\section{Introduction}

In-line metrology is a requirement of many high value manufacturing processes. It allows measurement data to be collected while a process is underway, or soon after it is completed. If measurements are made while the workpiece is in-situ, then corrective actions can be taken without needing to remove and re-fixture the workpiece. Robot deployed sensors offer a good way to make measurements in-situ. Systems such as robot- 
mounted 2D laser scanning systems offer a relatively low cost, highly flexible and robust way to measure a range of features. Robotic 2D laser scanning systems can be used in many industrial applications, such as: robotic machining [1],surface inspection [2-4], welding [5,6], belt grinding of aero-engines blades [7,8] or drilling operations [9].

The combination of a 2D laser sensor and a robot provides a highly versatile measurement system. The robot can be used to position the 2D laser sensor with high levels of control. This allows larger complex free-form surfaces and components to be easily inspected at a range of locations. It also makes 3D surface reconstruction possible by combining multiple measurements taken while moving the $2 \mathrm{D}$ laser sensor over the surface of the target object. However, to reconstruct data points in $3 \mathrm{D}$, the $3 \mathrm{D}$ homogeneous transformation between the sensor's coordinate system and the coordinate system of the robot tool flange is required. If this transform is not known, then data collected by the sensor at multiple positions cannot be transformed into one global coordinate system. When a vision system is mounted on a robot arm, the process of determining the homogeneous transformation between the robot tool-flange and the vision system's coordinate system is known as hand-eye calibration.

Hand-eye calibration methods are generally based on homogenous transformation equations of type $\mathrm{AX}=\mathrm{XB}$ [10-13]. For camera-based vision systems the hand-eye calibration problem is considered to be solved, with typical hand-eye calibration methods making use of checker board artefacts. Calibration algorithms make use of threedimensional data ( $\mathrm{x}$ and $\mathrm{y}$ pixel position, plus pixel intensity) that can be gathered from a camera image. In the case of 2D laser sensors, there is currently no widely adopted standard calibration strategy. The main issue lies in the fact that 2D laser sensors only provide two-dimensional data. Extracting enough data for calculating the six degree of freedom pose of the laser sensor therefore requires either the use of multiple measurements, the use of a 3D structured artefact or a combination of both.

For laser sensors the hand-eye calibration to determine the full homogenous transform including translation and rotation can be found in either a one-step or two-step process. In the two-step process rotation and translation are determined separately [14-17]. One drawback of the two-step calibration strategies is that calibration errors of the first step are propagated into the second step [18]. The two-step process is also inherently more complex; hence the trend is towards a simple one-step process. This is important as in many applications simplicity of the calibration process is a key benefit. Simplicity in this context can be considered in terms of the simplicity of the calibration object, and the simplicity of the required manual user interactions. These are important as they relate to the time and cost of the calibration procedure. For 2D laser sensors, existing calibration approaches tend to address one area of simplicity only. For example, the method proposed in [19] is based on visually positioning a laser profile sensor at a fixed Xshaped marker in different robot poses. This requires skilled manual interactions from the user, although the calibration object is still relatively simple. On the other hand, there are many examples of more complicated artefacts being used, such as a ball shaped object [14-17,20-22], disk shaped objects [23], objects with multiple stepped surfaces [24] or pin shaped objects [25]. The advantage of the more complex artefacts is that mostly the calibration is performed automatically, meaning the user interaction requirements are greatly simplified; however, the increased complexity of the artefact means it is harder to manufacture and more expensive. The calibration approach taken in this paper has been 
designed to provide simplicity of both the calibration object and the required user interactions; to satisfy this requirement it uses the most basic possible form of calibration object, which is a simple planar surface.

In previous work by Wei and Hirzinger [26] planar surfaces were used for finding the 3D positional and directional vectors of a laser diode range finder; using a single plane that was positioned in 13 unknown positions and orientations, with a manually controlled specific angle between the laser direction and normal vectors of each plane position. More recently, a robust hand-eye calibration strategy using random scan lines acquired from three close to orthogonal planes was suggested for robot mounted laser sensors [27]. The three-plane approach is attractive as there are no exact positioning constraints, allowing laser line scans to be collected from random positions on each or the three orthogonal planes. The use of random line scans makes this approach simple to setup, and the use of three orthogonal planes ensures enough data variation to allow the calibration problem to be solved using a linear Least Squares approach.

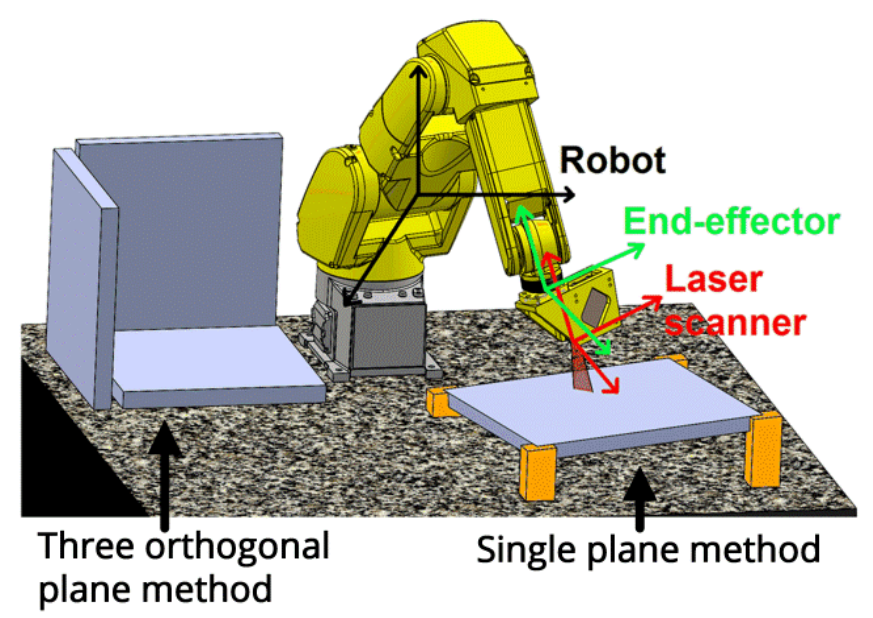

Figure 1. Illustration of a robot-mounted laser sensor system; three-plane setup versus a single-plane setup for hand-eye calibration.

The three-plane approach has two important drawbacks. First, careful interaction and control from the robot operator is needed to position the laser sensor relative to the three planes. Second, the need for three orthogonal planes introduces additional complexity to the process; for example, a single plane must be repositioned manually (by an operator) during the calibration procedure, or a more expensive and intrusive artefact with three orthogonal planes must be used. To address these issues, in this work an inherently simple hand-eye calibration strategy is proposed. This new method requires only a single flat surface for calibration. To illustrate the difference between the two approaches, a robotic laser sensor system located with the two sets of calibration artefact is shown in Figure 1. In addition, in order to simplify the physical implementation, the proposed calibration method can be used as part of a semi-automatic calibration process that requires manual collection of only four initial scan lines. 
In this work, the practicalities of using the single-plane strategy are considered in detail, and the calibration method is optimised for a robot mounted laser sensor system. A key contribution of the work is a methodology to define a suitable set of calibration data, that avoids non-convergent solutions, or solutions that converge to an erroneous result. The proposed methodology makes use of a circular pattern of target scan lines which are then used to define an associated set of robot poses to be used during the calibration. The most suitable robot poses are fully explored by simulating a robot mounted laser sensor to generate a large simulated data set, allowing a data driven optimisation approach. The paper is structured as follows. First, the theoretical basis for the single plane hand-eye calibration method is defined in Section 2. The simulation methodology and the process of using simulation data to determine suitable robot poses for a robust calibration are then described in Section 3. The potential performance of the single-plane calibration methodology is then assessed using simulation studies which are presented in Section 4. This is followed in section 5 by the results of physical experiments, which also demonstrate how the proposed calibration methodology can be executed as part of a semi-automatic calibration process. Finally, a discussion of the benefits of the method and the main conclusions drawn from this work are presented in sections 6 and 7 respectively.

\section{The theoretical basis for the single-plane calibration approach}

The Robot mounted laser sensor system shown in Figure 1 can be defined in terms of three coordinate systems: the robot base coordinated system, the end-effector coordinate system and the laser sensor coordinate system, as indicated on the figure. These three coordinate systems, along with parameters that define the orientation of the laser sensor relative to a target surface are illustrated in Figure 2, which defines the three main coordinate systems: the robot base frame coordinate system $\left(X_{R B}, Y_{R B}, Z_{R B}\right)$, the endeffector coordinate system $\left(X_{E F}, Y_{E F}, Z_{E F}\right)$ and the laser sensor coordinate system $\left(X_{S}, Y_{S}, Z_{S}\right)$. The objective of hand-eye calibration is to define the homogeneous transformation $\mathbf{T}_{\mathbf{E F}}^{\mathbf{S}}$ that describes the position of the sensor coordinate system relative to the end-effector coordinated system. Note in all mathematical descriptions used, boldface upper-case letters denote homogenous coordinate forms. The calibration process requires input data in the form of $2 \mathrm{D}$ profiles acquired by the laser sensor. Each of the $2 \mathrm{D}$ profiles vary based on the distance of the sensor to the central incident point $d$, and the orientation of the sensor with respect to the target line, shown in Figure 1 as the projection and tilt angles, $\theta$ and $\beta$ respectively.

The core equation of the plane-based hand-eye calibration approach is the transformation that relates any observed point in a $2 \mathrm{D}$ profile, $\mathrm{p}_{\mathrm{S}_{\mathrm{i}}}=\left[x_{S_{i}}, y_{S_{i}}, z_{S_{i}}\right]^{T}$ to its corresponding point on the calibration plane $\mathrm{p}_{\mathrm{RB}_{\mathrm{i}}}$; note the subscript $i$ denotes the index of the pose. This is performed using Equation 1 that requires the homogeneous $3 \mathrm{D}$ transformation from the robot to the end-effector, $\mathbf{T}_{\mathbf{R B}}^{\mathbf{E} \mathbf{F}_{\mathbf{i}}}$, and the unknown hand-eye transformation $\mathbf{T}_{\mathbf{E F}}^{\mathbf{S}}$. 


$$
\mathbf{P}_{\mathrm{RB}_{\mathrm{i}}}=\mathbf{T}_{\mathrm{RB}}^{\mathrm{EF}} \mathbf{T}_{\mathrm{EF}}^{\mathrm{S}} \mathbf{P}_{\mathrm{S}_{\mathrm{i}}}
$$

To determine $\mathbf{T}_{\mathbf{E F}}^{\mathrm{s}}$, Equation 1 can be solved using a Least Squares frame work; however, to achieve a robust solution, enough variation in the calibration data must be provided to form a well-posed Least Squares problem, with a full-rank coefficient matrix. In the case of the three-plane strategy [27], this is achieved based on three sources of variations; these are the surface normal of three planes (e.g. vector $n$ in Figure 2), the position of the end-effector in the robot base coordinated system $\mathbf{T}_{\mathbf{R B}}^{\mathbf{E F}_{\mathbf{i}}}$ and the variation in the $2 \mathrm{D}$ data acquired by the laser sensor. The unknown hand-eye transformation $\mathbf{T}_{\mathrm{EF}}^{\mathrm{S}}$ can then be found based on a two-step iterative framework. Starting with an initial guess for the hand-eye transformation $\mathbf{T}_{\mathbf{E F}}^{\mathrm{S}}$, which can be found by physical measurement, the following steps are repeated until convergence.

\subsection{Step a}

The measured points from the acquired 2D profiles are transformed from the 2D sensor coordinate system into the 3D robot frame, using Equation 1. For the first iteration, this is done using the initial guess for the unknown hand-eye transformation. The transformed points of each plane are then used to compute an estimation of the normal unit vector of the respective plane, $\overline{\mathrm{n}_{\mathrm{j}}}, j=1,2,3$ (each plane is defined by the subscript $j$ ). This is performed using Principal Component Analysis (PCA) [28].

The obtained normal vector has unit length. To maintain the requirements for the plane equation used in the next step (see Equation 2), the normal vector length is changed. This is performed by computing the distance $l$ of the orthogonal line from the origin to the plane, which is in the same direction as $\overline{n_{j}}$; Considering $\mu_{j}, j=1,2,3$ as the centre of each planes' mass $\mathrm{p}_{\mathrm{RB}_{\mathrm{i}}}$, the distance $l$ can be calculated as the length of the projection of the vector $\mu_{j}$, onto the plane normal $\overrightarrow{n_{j}}, l=\left\|\bar{n}_{j}\left(\bar{n}_{j}{ }^{T} \mu_{j}\right)\right\|=\bar{n}_{j} \mu_{j}$ and encoded into the normal vector by letting $\left\|n_{\mathrm{j}}\right\|=l$. Then, the normal vectors for the set of three planes is defined as: $n_{j}=\overline{n_{j}}\left(\bar{n}_{j}^{T} \mu_{j}\right), j=1,2,3$.

\subsection{Step $b$}

Knowing the estimate of the surface normal from the first step, it is possible to estimate a new hand-eye calibration transformation. This is done by fitting the transformed points in Equation 1 into a 3D plane equation. The equation of a 3D plane can be formed using the surface normal, one point in the plane and the distance $l$ (see Equation 2); $l$ can be obtained by dot product of the known point vector $\left(\mathrm{p}_{\mathrm{RB}_{\mathrm{i}}}\right.$ in this case) and the unit length 
normal vector, and it is encoded as the length of the normal vector $n_{j}$ as explained in step a, such that $\left\|\mathrm{n}_{\mathrm{j}}\right\|=l$.

$$
{ }_{\mathrm{n}_{\mathrm{j}}}^{-\mathrm{T}} \mathrm{p}_{\mathrm{RB}_{\mathrm{i}}}=l \leftrightarrow \mathrm{n}_{\mathrm{j}} \mathrm{p}_{\mathrm{RB}}=\left\|\mathrm{n}_{\mathrm{j}}\right\|^{2}, j=1,2,3
$$

Since the sensor acquires a 2D profile, then, $\forall i, y_{i}=0$ and without loss of generality, the points $\mathrm{p}_{\mathrm{S}_{\mathrm{i}}}$ can be assumed to lie on the plane $y_{S}=0$. As a result, the second column in $\mathbf{T}_{\mathrm{EF}}^{\mathrm{S}}$ cannot be solved directly. However, using the orthogonality constraints for the rotation matrix, $\mathrm{R}_{\mathrm{EF}}^{\mathrm{S}}$, the second column in $\mathrm{R}_{\mathrm{EF}}^{\mathrm{S}}$ can be computed by a cross product of the first and last columns. Let $\tilde{T}$ denote the remainder of $\mathbf{T}_{\mathbf{E F}}^{\mathrm{S}}$ after removing the second column and the last row. Then, Equation 1 and Equation 2 can be combined to yield Equation 3, where $\mathrm{w}=\operatorname{vec}(\tilde{\mathrm{T}}) \in \mathbb{R}^{9 \times 1}$ consists of the stacked columns of $\tilde{\mathrm{T}}$ and $\mathrm{A}_{\mathrm{i}}=$ $\left[\begin{array}{lll}\mathrm{n}_{\mathrm{i}}^{\mathrm{T}} \mathrm{R}_{\mathrm{RB}}^{\mathrm{EF} \mathrm{X}_{\mathrm{S}_{\mathrm{i}}}} & \mathrm{n}_{\mathrm{i}}^{\mathrm{T}} \mathrm{R}_{\mathrm{RB}}^{\mathrm{EF} \mathrm{Z}_{\mathrm{S}_{\mathrm{i}}}} & \mathrm{n}_{\mathrm{i}}^{\mathrm{T}} \mathrm{R}_{\mathrm{RB}}^{\mathrm{EF_{ \textrm {i } }}}\end{array}\right] \in \mathbb{R}^{1 \times 9}$ and $q_{i}=\mathrm{n}_{\mathrm{i}}^{\mathrm{T}} \mathrm{p}_{\mathrm{RB}}^{\mathrm{EF}} \in \mathbb{R}$.

$$
\mathrm{A}_{\mathrm{i}} \mathrm{W}+q_{i}=\left\|\mathrm{n}_{\mathrm{i}}\right\|^{2} \Leftrightarrow \mathrm{A}_{\mathrm{i}} \mathrm{w}=\left\|\mathrm{n}_{\mathrm{i}}\right\|^{2}-q_{i}
$$

Equation 3

Using the set of points from all three planes, a Least Squares solution can be used to find the first nine sets of parameters $\left(\mathrm{w}^{*}\right)$, as defined by Equation 4 , where $\mathrm{A}=\left[\mathrm{A}_{1}^{\mathrm{T}}, \ldots, \mathrm{A}_{\mathrm{N}_{\mathrm{P}}}^{\mathrm{T}}\right]^{\mathrm{T}}, \mathrm{Y}=\left[\left\|\mathrm{n}_{1}\right\|^{2}-q_{1}, \ldots,\left\|\mathrm{n}_{\mathrm{N}_{\mathrm{P}}}\right\|^{2}-q_{\mathrm{N}_{\mathrm{P}}}\right]^{T}$. The computed $\mathrm{w}^{*}$ only contains the first and last column of $\mathrm{R}_{\mathrm{EF}}^{\mathrm{S}}$ and the second column can be found based on their cross product, where $\mathrm{R}_{2}=\mathrm{R}_{3} \times \mathrm{R}_{1}$ so that $\mathrm{R}_{\mathrm{EF}}^{\mathrm{S}}=\left[\mathrm{R}_{1}, \mathrm{R}_{2}, \mathrm{R}_{3}\right]$.

$$
\mathrm{w}^{*}=\operatorname{argmin}_{\mathrm{w}}\|\mathrm{Aw}-\mathrm{Y}\|_{2}=\left(\mathrm{A}^{\mathrm{T}} \mathrm{A}\right)^{-1} \mathrm{~A}^{\mathrm{T}} \mathrm{Y}
$$

Equation 4

Singular Value Decomposition (SVD) is then applied on the resulting rotation matrix $\mathrm{R}_{\mathrm{EF}}^{\mathrm{S}}$, which gives the closest valid rotation matrix [29] belonging to $S O(3)$. Finally, since the procedure of orthogonalizing $\mathrm{R}$ will change the corresponding entries in $\mathrm{w}^{*}$, the resulting coefficients will no longer be valid for Equation 5. Therefore, the translational part of $\mathrm{w}$ is re-estimated by a second optimization. For this aim, $\mathrm{w}$ is decomposed, $\mathrm{w}=\left[\begin{array}{c}\tilde{\mathrm{R}}^{*} \\ \operatorname{Tr}\end{array}\right], \tilde{\mathrm{R}}^{*} \in \mathbb{R}^{6 \times 1}, \operatorname{Tr} \in \mathbb{R}^{3 \times 1}$. The rotation part of the target vector $\mathrm{Y}$ is 
ignored so that, $\tilde{\mathrm{Y}}=\mathrm{Y}-\mathrm{A}_{1: 6} \tilde{\mathrm{R}}^{*}$ ( $\mathrm{A}_{k_{1}: k_{2}}$ denotes the columns $k_{1}$ to $k_{2}$ of the matrix). Finally, the optimal translational vector is recomputed using Equation 5.

$$
\operatorname{Tr}^{*}=\operatorname{argmin}_{\mathbf{t}}\left\|\mathrm{A}_{7: 9} \operatorname{Tr}^{*}-\tilde{\mathrm{Y}}\right\|=\left(\mathrm{A}_{7: 9}^{\mathrm{T}} \mathrm{A}_{7: 9}\right)^{-1} \mathrm{~A}_{7: 9}^{\mathrm{T}} \tilde{\mathrm{Y}}
$$

Equation 5

Using the new estimated homogeneous transformation $\mathbf{T}_{\mathbf{E F}}^{\mathbf{S}}$, the two steps, a and $\mathbf{b}$, are repeated until the changes in the estimated transformation matrix is negligible.

In order to find the nine unknown parameters in Equation 4, a system of nine independent equations (coefficient matrix of rank nine) is required; however, the use of data from three orthogonal planes does not fulfil the rank condition. Therefore, more than nine equations are formed using data from several lines captured from each of the three planes. This increases the independency between the coefficient vectors of the nine variable, and achieves a full rank input matrix A to find a unique solution [28]. It also allows a linear Least Squares method to be used to find the unknown parameters as described in [27].

In transition from three orthogonal planes to a single-plane approach, significant care must be taken to ensure the calibration data includes the necessary variation required for the algorithm. Especially because the surface normal, which is one of the three original sources of variation in the linear set of equations (Equation 4), is not a variable in the single-plane strategy; in the single-plane approach only two sources of variations, $\mathrm{p}_{\mathrm{S}_{\mathrm{i}}}=\left[x_{S_{i}}, 0, z_{S_{i}}\right]^{T}$ and $\mathbf{T}_{\mathbf{R B}}^{\mathbf{E F}_{\mathbf{i}}}$ remain. In order to have clear insight about the role of these variation sources, the plane equation (Equation 3) is shown based on these parameters in Equation 6 and Equation 7. The surface normal is defined based on the plane orientation, i.e. the position that it is assigned during calibration. $\mathbf{T}_{\mathbf{R B}}^{\mathbf{E \mathbf { F } _ { \mathbf { i } }}}$ varies as a function of the robot's pose, and the $2 \mathrm{D}$ profile data measured by the laser sensor is affected by the scan parameters $(d, \theta$ and $\beta$ ) as shown in Figure 2. In Equation 6, the second element of the $2 \mathrm{D}$ profile $\mathrm{p}_{\mathrm{S}_{\mathrm{i}}}$ is line crossed since the laser sensor only acquires data in the $\mathrm{x}$ and $\mathrm{z}$ directions. Therefore, the second column elements in $\mathbf{T}_{\mathbf{E F}}^{\mathbf{S}}$ cannot be calculated so they are line crossed, but they can be found in a later stage by taking the cross product of the first and last columns.

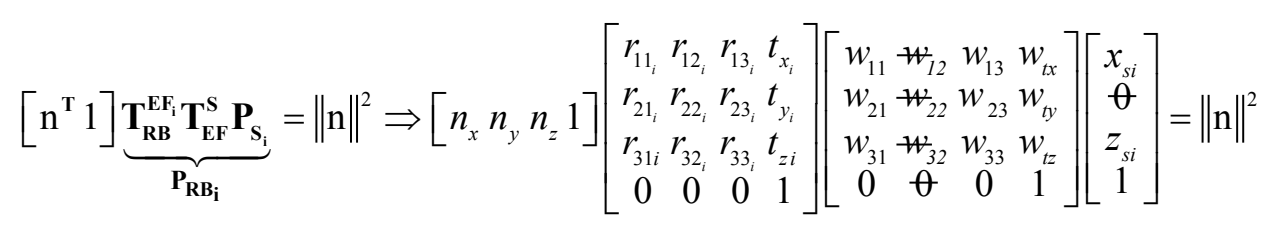




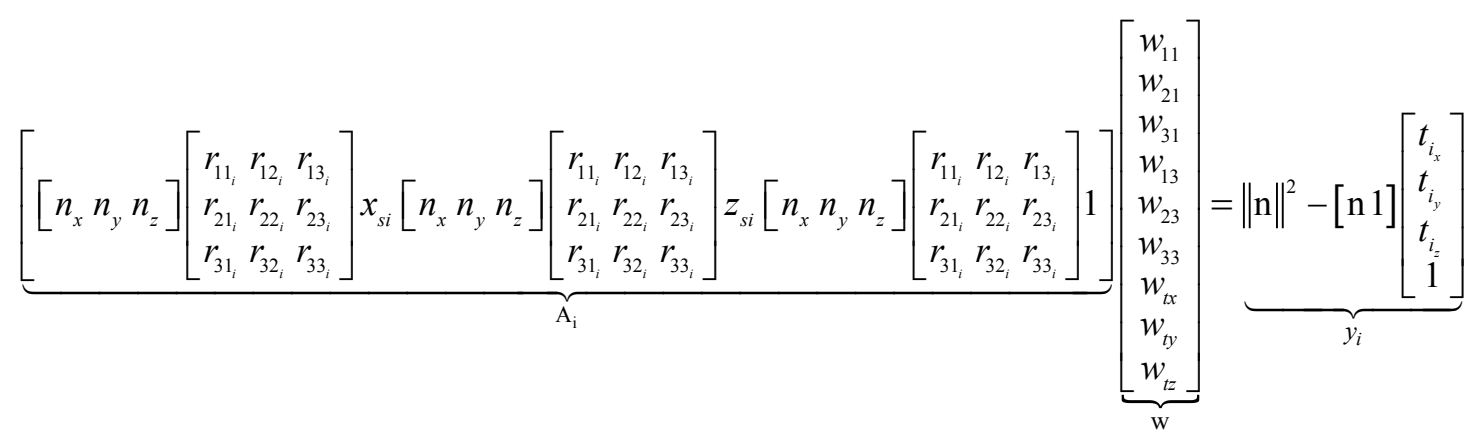

Equation 7

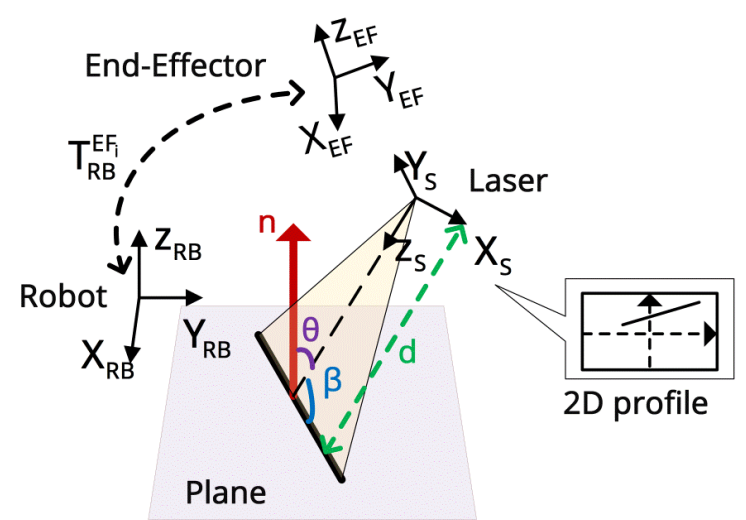

Figure 2. A laser sensor located at distance $d$ from the incident point. $n, \theta, \beta$ are the surface normal, projection angle and tilt angles respectively.

By increasing the variations of the two remaining sources, the laser sensor 2D profile $\mathrm{p}_{\mathrm{S}_{\mathrm{i}}}$ and the end-effector transformation $\mathbf{T}_{\mathbf{R B}}^{\mathbf{E F}_{\mathbf{i}}}$, it is possible to compensate for the lack of surface normal variation and form a full rank input matrix needed for a Least Squares solution. Regarding the variation in $\mathbf{T}_{\mathbf{R B}}^{\mathbf{E F}_{\mathbf{i}}}$, from Equation 7, it can be seen that the terms $n{ }^{T} R_{R B}^{E F_{i}}$ and $n{ }^{T} T_{R B}^{E F_{i}}$ can be varied by changing the robot pose when scanning successive lines. This maintains the variations between the equations of different lines (between-line variation). Regarding the variation of $\mathrm{p}_{\mathrm{s}_{\mathrm{i}}}$, given a fixed set of sensor parameters $(d, \theta, \beta)$, the same $2 \mathrm{D}$ profile $\mathrm{p}_{\mathrm{S}_{\mathrm{i}}}=\left[\begin{array}{lll}x_{i} & y_{i} & z_{i}\end{array}\right], y_{i}=0$, will be acquired for any robot pose. However, within a single $2 \mathrm{D}$ profile, there is slight variation in the equations of different points belonging to the same profile. The small variations are in their $x_{i}$ and $z_{i}$ values (within-line variation). These two sources of variations, the laser sensor 2D profile and the end-effector transformation, should be improved in terms of variation (maximised in the ideal case) to achieve a full rank input matrix A. However, there are some limitations in this case; while an equation can be formed for every observed point in a line, a high level of dependency exists between the equations of two close points in a line. Furthermore, depending on the directionality of the scanned lines, correlations might 
exist between their direction in the robot's coordinate system, resulting in low variations in robot arm movements and the resulting $\mathbf{T}_{\mathbf{R B}}^{\mathbf{E F _ { \mathbf { B } }}}$; this also causes correlations in their $2 \mathrm{D}$ profiles $\mathrm{p}_{\mathrm{s}_{\mathrm{i}}}$. To overcome this problem the number of scans must be increased, using various sensor parameters $(d, \theta, \beta)$. This results in additional variations from both the acquired 2D profiles $\mathrm{p}_{\mathrm{S}_{\mathrm{i}}}$ and the end-effector transformation $\mathbf{T}_{\mathrm{RB}}^{\mathbf{E F _ { \mathrm { i } }}}$. A natural result of increasing the orientation of the lines, scanned on the single plane calibration artefact, is that this will also tend to increase the variations in the movement of the robot arm.

In addition to considering how the sensor is positioned during calibration, it is also necessary to consider where the calibration plane is positioned relative to the robot. This is because an important potential limiting factor happens when there are zero elements in the surface normal $\mathrm{n}$ of the plane. This happens when the plane orientation is parallel to one of the $X Y, X Z$ or $Y Z$ planes. In this condition, two of the three elements of the normal vector $\mathrm{n}$ are zero; tending to nullify the effect of their multiplied rows in $\mathrm{R}_{\mathrm{RB}}^{\mathrm{EF}}$ in Equation 7 , and the only remaining row in $\mathrm{R}_{\mathrm{RB}}^{\mathrm{EF}}$ is similar for all the data lines in that plane, regardless of their position and orientation. This is due to the fact that there is no variation along the non-zero axis. For example, for a plane with the rotation orientation defined by $\theta_{x}=0, \theta_{y}=0, \quad \theta_{z} \neq 0$, then the normal vector is $\mathrm{n}=\left[\begin{array}{lll}0 & 0 & 1\end{array}\right]^{T}$. Using the same scanning parameters $(d, \theta, \beta)$, the equations of any line acquired from any position in the plane is similar; that is, both the remaining row $\left[\begin{array}{lll}r_{31_{i}} & r_{32_{i}} & r_{33_{i}}\end{array}\right]$ and the $2 \mathrm{D}$ profile $\mathrm{p}_{\mathrm{S}_{\mathrm{i}}}$ are the same for all of the acquired profiles (see Equation 8). In other words, if the sensor position parameters are fixed there is no benefit in scanning additional lines from the surface in this condition.

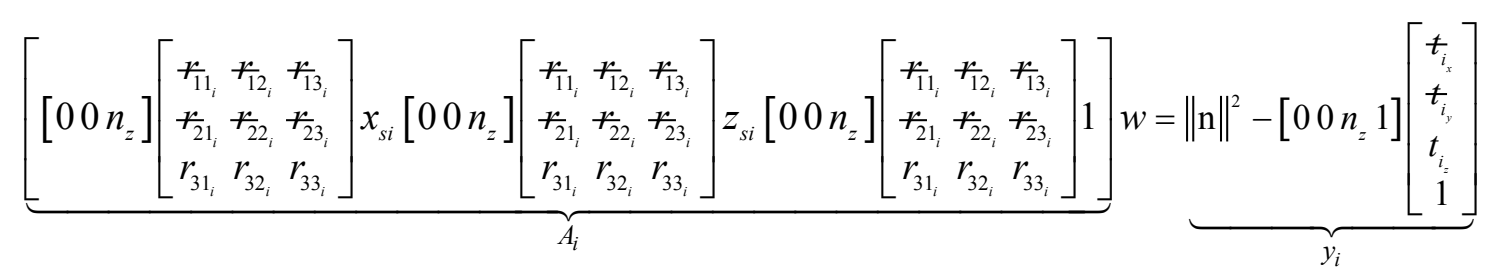

Equation 8

The only remaining sources of variation in this condition are the sensor parameters influencing the $2 \mathrm{D}$ profile $\mathrm{p}_{\mathrm{S}_{\mathrm{i}}}$. Due to the correlations of the lines in $2 \mathrm{D}$ profile and the resulting dependencies in the equations, it is difficult to maintain a full rank input and find a unique solution for the hand-eye calibration problem. Therefore, such a condition should be avoided, and this will be further investigated by an optimisation study presented in Section 4. 


\section{Measurement system simulation for calibration process evaluation}

To investigate the impact of various strategies for collecting calibration data, a general simulation of a robot mounted laser sensor was created. The simulation model considers four key aspects: the position of the plane relative to the robot, the position of the sensor relative to the robot's end-effector, the pattern of scan lines acquired from the target plane and the reach of the robot. Within the simulation, the nominal hand-eye calibration transformation, a set of reach constraints for the robot, and the position of the single plane calibration artefact in the robot's coordinate frame are specified. The position of the artefact is defined in terms of the angular rotations the lateral translations of the artefact with respect to the origin of the robot's base frame. Furthermore, a set of target lines that will be acquired by the laser sensor, are defined on the surface of the artefact. Two strategies are considered to define the target lines, they can be either randomly distributed, or they can be defined based on a predetermined pattern. Within the simulation provision was made to accommodate both strategies; allowing data representative of a set of random lines, as well as a predefined circular pattern, to be generated. The random pattern is defined using a computer random number generator, while the circular pattern consists of nine lines, each with one end located at a coincident point at the centre of a circle, and the other end of each line spaced equally on the circumference of the circle, such that there is a 40 degrees angle from one line to the next. The circular pattern is designed to maximise the rotational variation from a single $2 \mathrm{D}$ plane between the target lines. It also provides a simple starting point from which to generate a full set of calibration data, which is useful for automation of the process. Figure 3 shows examples of the two strategies.

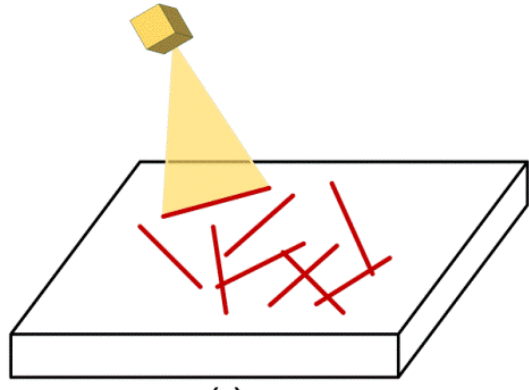

(a)

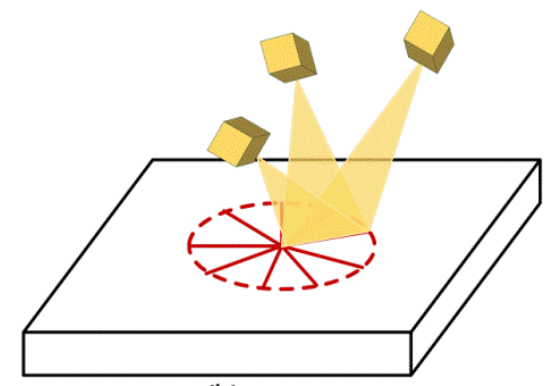

(b)

Figure 3. Illustration of the two strategies for defining the target lines; (a) random distribution (b) circular pattern.

To ensure sufficient variation is included in the simulated data set, for each target line a set of 3D sensors poses are also considered. The set of sensor poses represent different positions of the sensor relative to the calibration plane such that it can measure a set of points from the same target line. The pose of the sensor relative to the target line is represented by the three scan parameters, $d, \theta$ and $\beta$ as shown in Figure 2. For each combination of these parameters a sensor pose in the robot coordinate system is defined, and from this the corresponding 2D laser profile and the position of the robot tool flange $\mathbf{T}_{\mathbf{R B}}^{\mathbf{E \mathbf { F } _ { \mathbf { i } }}}$ is simulated. To ensure that each sensor pose is feasible the pose is checked to ensure it lies within estimates of reachability for the robot arm. 


\section{Performance assessment of the single-plane hand-eye calibration process}

A major advantage of the simulation approach is that it allows many different calibration configurations to be easily compared. Also, as the hand-eye calibration is known in the simulation, it provides a useful means to evaluate the effect of data variability and quality on the accuracy of the calibration process.

\subsection{Hand-eye calibration for multiple robot systems}

In order to evaluate the single-plane hand-eye calibration strategy on different configurations of laser sensor systems, 200 sensor systems with different hand-eye calibration matrices were simulated. The translational parameters of hand-eye calibrations matrices for each system were chosen from a uniform distribution $U(-100 \mathrm{~mm},+200 \mathrm{~mm})$ and similarly, the angular parameters were distributed uniformly $U\left(-180^{\circ},+180^{\circ}\right)$. For each simulated system, a single plane, oriented randomly in front of the robotic system, was considered. The rotation angles of the planes around $x, y$, and $z$ directions within the robot coordinate system were constrained to be not close to zero with all rotations greater that one degree. This was done to avoid the problems related to small angles explained in Section 2.

A circular pattern of nine lines was simulated on each of the planes. Based on these lines a wide range of $2 \mathrm{D}$ scan profiles in the laser sensors coordinate system was calculated. The 2D Scan profiles were based on sensor position parameters that define the pose of the sensor relative to the target lines, i.e. projection angle and tilt angle as shown in Figure 2. The projection angle $\theta$ was varied in the range $0^{\circ} \leq \theta \leq 40^{\circ}$, with a step size of five degrees. The tilt angle $\beta$ was varied in the range $60^{\circ} \leq \beta \leq 120^{\circ}$, with a step size of ten degrees and the height parameter $d$ was varied within the range of $60 \mathrm{~mm} \leq d \leq 120 \mathrm{~mm}$, with a ten mm step size. 2D profiles were simulated for all of the nine lines based on all possible combinations of the scan parameters, under the condition that the robot reachability constraints were fulfilled. The corresponding $\mathbf{T}_{\mathbf{R B}}^{\mathbf{E F _ { \mathbf { i } }}}$ transformations were also computed in each case.

Using the simulated data for each system, hand-eye calibration was performed. The initial guess for the hand-eye calibration algorithm was generated in each case by adding a uniformly distributed offset, ranging plus and minus ten percent of nominal, to each of the values in the true hand-eye calibration. Figure 4 shows box plots that illustrate the distribution of errors in the achieved hand-eye calibrations for all these experiments. As can be seen the errors are very low, showing that even with a single plane, as long as good variation in the calibration data is maintained, it is possible to achieve good calibration results. 

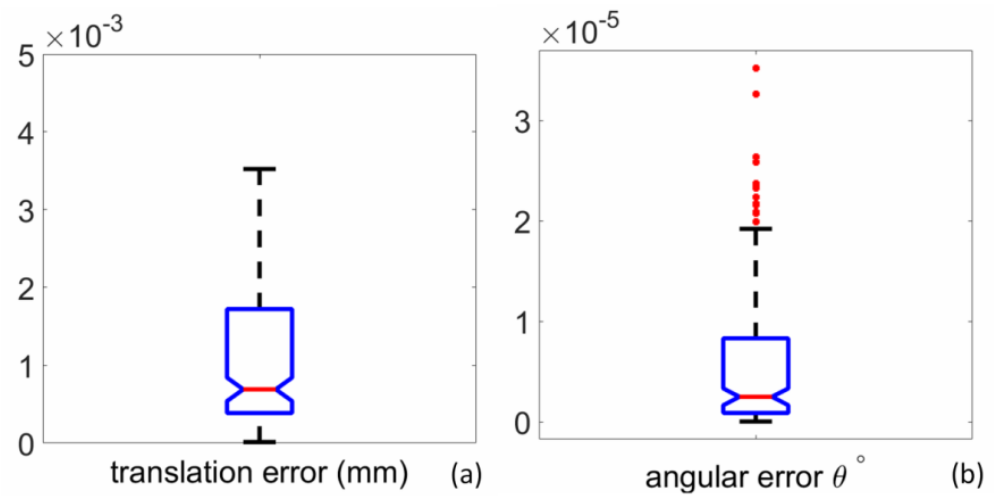

Figure 4. The average error results of single-plane hand-eye calibrations, using 200 different simulated systems (a) translational elements (b) rotational elements. For ease of visualisation, five outliers all with translational errors smaller than $0.03 \mathrm{~mm}$ and angular errors smaller than $0.5 \times 10^{-4}$ degrees are not included.

\subsection{Hand-eye calibration process optimisation}

To maintain good variation in the calibration data large quantities of calibration data can be used, however for practical reasons, reducing the number of scans required for calibration is an obvious benefit. Given a real robot-mounted laser sensor, the hand-eye calibration should be optimised to minimize the required number of scans for successful calibration. The simulation was therefore used to investigate how the calibration data set can be reduced while still maintaining robust calibration results. The three main factors affecting data variation are the plane orientation, number of lines scanned and the sensor position parameters $(d, \theta, \beta$, see Figure 2). Each of these factors is considered in the following three sub-sections.

Over the simulations, the plane orientation angles $\left(\alpha_{x}, \alpha_{y}, \alpha_{z}\right)$ are varied in the range of $\left(-5^{o} \leq \alpha \leq 5^{\circ}\right)$ with a step size of $1^{\circ}$. The reason for the choice of this range is the closeness to $0^{\circ}$ which is easier and simpler to maintain in practice. All the $11^{3}$ possible combinations of the three angles are considered. For each plane orientation a circular pattern of nine lines is simulated on the plane. Then, based on the sensor position parameters $(d, \theta, \beta)$, the $2 \mathrm{D}$ profiles $\mathrm{p}_{\mathrm{S}_{\mathrm{i}}}$ as well as $\mathbf{T}_{\mathbf{R B}}^{\mathrm{EF}_{\mathrm{i}}}$ transformations for each line are simulated. The distance is varied in range $d \in\left[\begin{array}{lll}60 \mathrm{~mm} & 90 \mathrm{~mm} & 120 \mathrm{~mm}\end{array}\right]$ which was selected based on the physical focal range of the selected laser sensor. The projection angle is chosen to provide one extreme and one middle value such that $\theta \in\left[\begin{array}{ll}0^{\circ} & 30^{\circ}\end{array}\right]$. The tilt angle is varied in three values $\beta \in\left[\begin{array}{lll}60^{\circ} & 90^{\circ} & 120^{\circ}\end{array}\right]$. It must be noted that the number of generated 2D profiles for each line is less than the full set of possible combinations of $d, \theta$ and $\beta$. This is due to the fact that some of the found sensor poses are estimated to be outside the reachable boundaries of the robot arm. Hence, these nonconforming cases are ignored. 


\subsubsection{Orientation of the plane}

The plane orientation influences the surface normal, $n$, which appears in the hand-eye calibration equation (see equation 6). Hand-eye calibration was performed for all of the $11^{3}$ simulated planes explained in section 4.2. Only in 15 out of the total of $11^{3}$ cases, the calibration was unsuccessful. A calibration is considered unsuccessful, when after many iterations (above than an upper threshold of 2000), the algorithm did not converge or it converged before the upper threshold but, the deviation from the true hand-eye calibration is more than $0.01 \mathrm{~mm}$ in at least one of the elements. Table 1 shows the number of successful and unsuccessful cases based on the number of plane orientation angles, $\left(\alpha_{\mathrm{x}}, \alpha_{\mathrm{y}}, \alpha_{\mathrm{z}}\right)$ that are close to zero $\left(-1^{\circ} \leq \alpha_{i} \leq 1^{\circ}\right)$. The occurrences vary from zero (cases without any close-to-zero angles) to three (when all of the plane orientation angles are close to zero). As can be seen, in unsuccessful cases at least one of the three angles was close to zero. On the other hand, there were no unsuccessful cases when all of the three angles were outside the range $-1^{\circ} \leq \alpha_{i} \leq 1^{\circ}$. This result supports the mathematical reasons explained for these problematic angles in Section 2. Hence, the use of non-zero angles is considered as a criterion for orientation of the single plane for robust calibration.

Table 1. Number of successful and unsuccessful calibration cases based on the number of plane orientation angles $\left(\alpha_{\mathrm{x}}, \alpha_{\mathrm{y},} \alpha_{\mathrm{z}}\right)$ that is close to zero $\left(-1^{\circ} \leq \alpha_{i} \leq 1^{\circ}\right)$.

\begin{tabular}{lll}
\hline$-1^{\circ} \leq \alpha_{i} \leq 1^{\circ}, i=x, y, z$ & Number of Succ. calib. & Number of Unsucc. calib. \\
\hline 0 & 512 & 0 \\
1 & 573 & 3 \\
2 & 208 & 8 \\
3 & 23 & 4 \\
\hline
\end{tabular}

\subsubsection{Laser sensor positional parameters}

In order to minimise the number of values for scan parameters, a top down strategy is considered. Therefore, the success of hand-eye calibration for reduced cases of the candidate values for each scan parameter is investigated for all appropriate plane orientations. This process continues for a lower number of values until a failed calibration case occurs. The search stops when a failed calibration is found since it contradicts the initial aim for finding a generalised solution that works for any appropriate plane orientation.

Starting with the laser height $(d)$, one of the three candidate height values is ignored each time. In each of the three resulting cases, all possible combinations of the eight appropriate values for $\left(\alpha_{\mathrm{x}}, \alpha_{\mathrm{y}}, \alpha_{\mathrm{z}}\right)$ is considered and all the candidate values of the two other parameter's $(\theta, \beta)$ are used. This results in 1536 data sets, however the hand-eye calibration failed in 26 cases out of the 1536 total tests.

Table 2 shows the number of successful and unsuccessful cases based on the ignored heights. As can be seen, in most unsuccessful cases, the failure happened due to ignoring the $2 \mathrm{D}$ profiles with the laser sensor at the closest distance to the scanned lines, while ignoring the furthest distance of $120 \mathrm{~mm}$ resulted in only two failed calibrations. 
Although there is a low risk of failure when ignoring $120 \mathrm{~mm}$, keeping all of the three heights is necessary to avoid the risk of failed calibration.

Table 2. The number of successful and unsuccessful calibration cases based on the ignored heights of the sensor $(d)$.

\begin{tabular}{lll}
\hline Ignored Height & Number of Succ. calib. & Number of Unsucc. calib. \\
\hline $60 \mathrm{~mm}$ & 494 & 18 \\
$90 \mathrm{~mm}$ & 506 & 6 \\
$120 \mathrm{~mm}$ & 510 & 2 \\
\hline
\end{tabular}

Similarly, in the case of projection angle $\theta$, the ranges of variation are considered and based on the top-down strategy, the effect of elimination of one of the two initial candidate values from the set is evaluated. This is performed for the appropriate plane orientations and the three height variables and all the candidate $\beta$ values were used, resulting in $8^{3} \times 2=1024$ data sets for calibration. Half of the calibrations failed, and the other half were successful; in summary, ignoring the $30^{\circ}$ projection angle caused failure while ignoring the $0^{\circ}$ resulted in successful calibrations. The reason for this can be explained by considering the illustration in Figure 2, at an orthogonal projection angle $(\theta$ $\left.=0^{\circ}\right)$, the tilt angle becomes orthogonal $\left(\beta=90^{\circ}\right)$; in this case the variations between different acquired 2D profiles are reduced significantly. Based on these results, the use of one projection angle, $\theta=30^{\circ}$ is deemed enough for hand-eye calibration.

The same top-down strategy is also used for the tilt angle $\beta$. All combinations of the two out of the three candidate tilt angles $\beta$ are considered together with all heights and only one projection angle, $30^{\circ}$, resulting in a total of 1536 datasets. There were 98 failed calibrations and 1438 successful cases. Table 3 shows the number of successful and unsuccessful calibration cases based on ignored angles. As can be seen, ignoring any of the three angles resulted in failed cases, so all the three tilt angles are recommended calibration.

Table 3. Number of successful and unsuccessful calibration cases based on ignored tilt angles $(\beta)$.

\begin{tabular}{lll}
\hline Ignored Angle & Number of Succ. calib. & Number of Unsucc. calib. \\
\hline $60^{\circ}$ & 466 & 46 \\
$90^{\circ}$ & 469 & 43 \\
$120^{\circ}$ & 503 & 9 \\
\hline
\end{tabular}

\subsubsection{Number of lines}

Hand-eye calibration using the sensor position parameters for the nine lines of the circular pattern and the appropriate plane orientations $\left(\alpha_{x, y, z}<-1^{\circ}\right.$ and $\left.\alpha_{x, y, z}>1^{\circ}\right)$, achieved successful results. To minimize the number of required lines, the top-down strategy was used again, and the number of target lines was reduced from nine to eight. This resulted in six cases of failed calibration. Therefore, it is recommended that all nine lines in the circular pattern should be used for a successful calibration. 
Furthermore, to investigate the possibility of using lines randomly, and compare this to the circular pattern approach, many calibration tests were performed over sets of simulated data. 500 sets of random lines were simulated on a plane oriented each time randomly using the range of appropriate rotations $\left(\alpha_{\mathrm{x}}, \alpha_{\mathrm{y}}, \alpha_{\mathrm{z}}\right)$. Totally 81 random lines are simulated on each plane. 81 lines was chosen to mirror the 9 lines in circular pattern experiments, where for each line there are 9 possible combinations of the optimal parameters (one $\theta$, three heights and three $\beta$ values). Hand-eye calibration was performed successfully on all of the 500 simulated sets. The averages and standard deviations of the translational and angular errors were $(0.0435 \pm 0.269) \times 10^{-3} \mathrm{~mm}$ and $\left(0.060^{\circ} \pm 0.237^{\circ}\right) \times 10^{-3}$ respectively, with the value before the \pm symbol giving the mean and the value after giving the standard deviation.

\subsection{Simulating the effect of imperfect data}

To investigate the impact of imperfect data on the single-plane calibration process, data points within each simulated 2D profile are modified by the addition of a noise term. The noise is modelled using a Gaussian function with zero mean. The standard deviation of the Gaussian is then varied to simulate a range of noise levels. The range of values chosen was based on noise characterisation experiments of the real set-up, that were performed previously [30,31], and the level of noise that was considered previously for evaluation of the three-plane hand-eye calibration algorithm [27].

As the single-plane hand-eye calibration algorithm is based on minimization of a Least Squares equation as shown in Equation (4), increasing the number of scanned lines will provide more data for fitting and help to cancel out the effect of noise. To determine the potential benefits of increasing the number of scanned lines, for each level of noise considered, the number of simulated scanned lines is also varied. Eight sets of simulated lines are considered, with the quantity of lines in each set nominally set to $81,180,270$, $360,450,540,900$ and 4500 . These nominal quantities are based on random sets of target lines, that must be scanned on the calibration surface (lines set sizes: 9, 20, 30, 40, 50,60, 100 and 150), with each target line scanned nine times based on all nine optimal scan parameters, as defined above. Due to the random distributions of target scan lines, as described previously, based on the robot arm reachability limits some lines were discounted so the actual number of scans can sometimes be slightly less than nine per target line.

For each nominal quantity of lines, the simulations were repeated 100 times, and each time the lines were located on a single plane that was oriented randomly but was in an appropriate orientation as defined above. Gaussian noise in different standard deviation levels ranging in $0 \leq \sigma_{\text {noise }} \leq 0.5 \mathrm{~mm}$ with the step size of $0.05 \mathrm{~mm}$ was added to the simulated points in each scanned line. The first case, with zero standard deviation is a clean signal condition.

Furthermore, in order to compare the single-plane hand-eye calibration strategy with the three-plane strategy [27], simulations of data from three planes was also performed. One hundred sets of data were created in which a plane was simulated randomly, and two other orthogonal planes were then also simulated in positions relative to the first plane. For these experiments 35 random lines were simulated on each plane and the associated 
sensor poses, 2D profiles $\mathrm{p}_{\mathrm{S}_{\mathrm{i}}}$ as well as the transformation $\mathbf{T}_{\mathbf{R B}}^{\mathrm{EF}}$ data were simulated. The same set of Gaussian noise levels were added to these data sets giving totally 1100 sets of data each consisting of $3 \times 35=105$ scan lines.

Hand-eye calibration was performed for all the simulated data sets. In all cases, the initial guess for hand-eye calibration was formed by adding a large level of uniformly distributed noise to the true hand-eye elements; this is defined as $U\left(-30^{\circ},+30^{\circ}\right)$ for the angular elements and $U(-200 \mathrm{~mm},+200 \mathrm{~mm})$ for the translational elements. The results of the calibrations cab be seen in Figure 5, which shows the averages of translational and rotational errors over the 100 simulations for all single-plane as well as three-plane data sets. The $X$ axes show the average number of used lines and the $Y$ axes shows the noise level. As can be seen both errors reduce when the number of lines increases, and they are also smaller in cleaner signal conditions. A linear curve fitting strategy is used to compute the intermediate points (between the measured points) in the plot. The threeplane calibration results, consisting of 105 lines, are also included as a line of dots on the surface plot shown in Figure 5. As the dots are coincident with surface plot, the calibration results for three-plane calibration are consistent with the errors expected for a similar quantity of lines acquired from a single plane. In general it can also be seen that in noisier conditions, the use of more lines can reduce the calibration errors. 

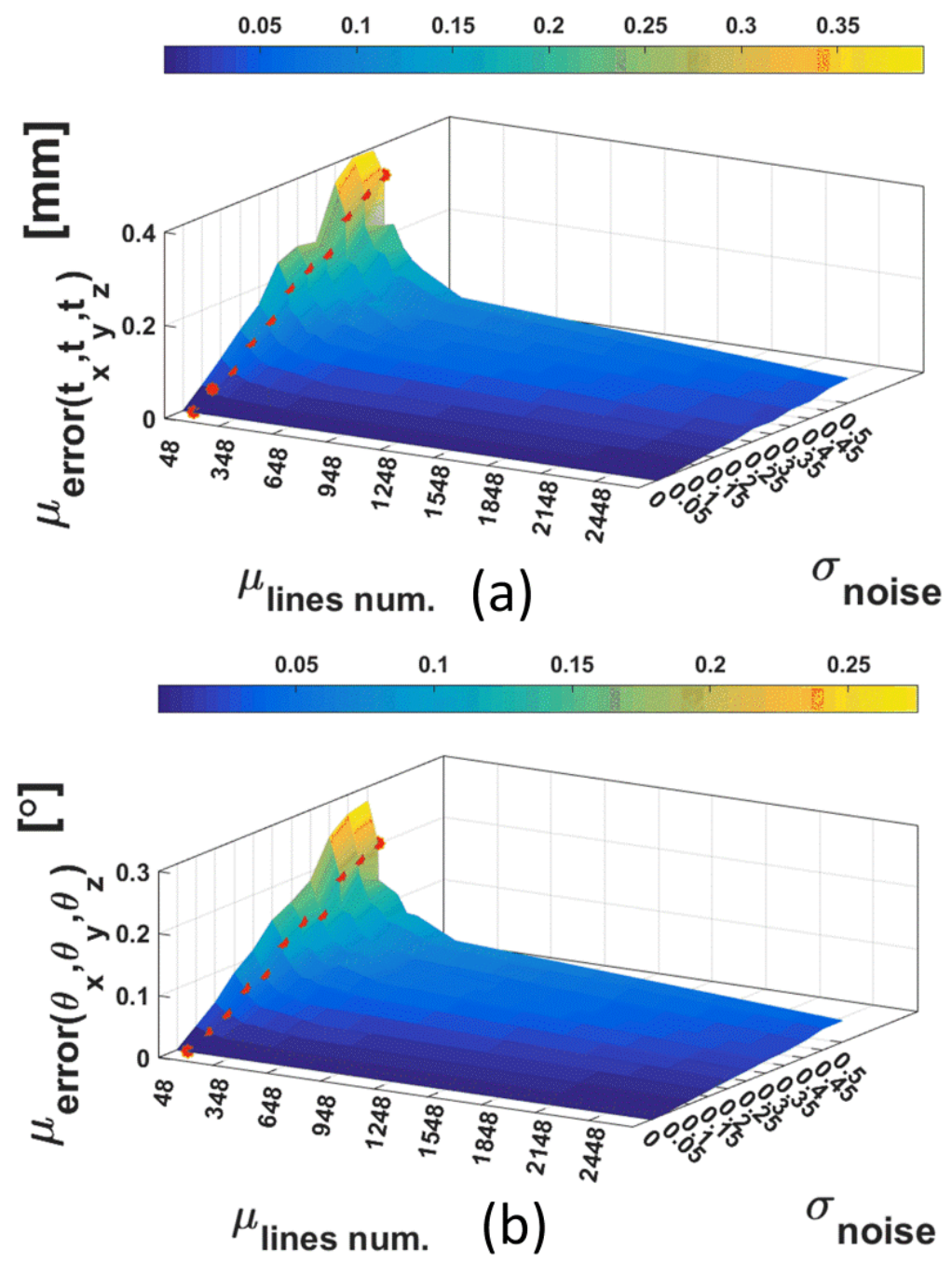

Figure 5. The average hand-eye calibration errors, for different quantities of scanned lines and Gaussian noise levels, over 100 simulations per line number and noise level, (a) translation error (b) angular error. The results of the three-plane strategy using 105 lines are over plotted in dots.

\section{Physical validation of the single-plane hand-eye calibration process}

To validate the proposed single-plane calibration strategy, four sets of data were collected [32]; one based on the previous three-plane method [27], one based a systematically generated data from the circular pattern of nine lines, that resulted from the simulation studies defined in section 4 , one based on a computer randomized pattern and one based on a random selection of manually defined robot poses as defined by the robot operator. The robot mounted laser sensor system used to collect this data is shown in Figure 1. The robot is a Fanuc LR Mate $200 \mathrm{iC}$ industrial robot arm, driven by a R30/iA Mate controller. The sensor is a Micro Epsilon 3D profile sensor (sensor model: Epsilon scan control 2900-50); it is a commercial laser sensor, consisting of a laser light source, a sensor matrix and a receiver. All data was collected from a flat rectangular surface artefact of $400 \mathrm{~mm} \times 500 \mathrm{~mm}$ x $25 \mathrm{~mm}$. The flatness of the target surface on the 
artefact was measured using a Nikon Ultra CMM, probing an evenly distributed array of 357 points over the surface. In this way the surface artefact was found to have a total flatness variation of $+/-21 \mu \mathrm{m}$.

For the first set of data that replicates the three-plane method [27], the artefact was positioned in three almost orthogonal planes and 35 random lines were collected from each of the three plane positions. The scans were repeated 5 times per plane, with the robot visiting the same poses each time. Using this data set, hand-eye calibration was performed using 125 different combinations of the acquired data, resulting in 125 calibration matrices.

For the second set of data, the plane artefact was located approximately $410 \mathrm{~mm}$ in front of the robot and $150 \mathrm{~mm}$ to the robots right hand side, and it was tilted at an angle of approximately five degrees to the $\mathrm{x}, \mathrm{y}$, and $\mathrm{z}$ axis of the robots coordinated system; for this aim, four holders were designed and 3D-printed as was shown in Figure 1. Based on the ideal set of circular lines and associated sensor poses, that was determined by the simulation work, a reduced set of 48 sensor poses was selected for testing. Using the set of 48 sensor poses, the robot was cycled through the poses five times allowing the collection of five data sets and consequently five calibration matrices.

For the third set, again the plane was located approximately $410 \mathrm{~mm}$ in front of the robot and $150 \mathrm{~mm}$ to the robots right hand side, and it was tilted at an angle of approximately five degrees to the $\mathrm{x}, \mathrm{y}$, and $\mathrm{z}$ axis. Data was collected from a set of 105 randomly generated sensor poses that were defined by computer, and the sequence of scans was repeated five times. In Figure 6, the simulated data as well as the data collected by the sensor are visualised on the same plot. This represents the second and third data sets, and as can be seen, the simulated and real data are well aligned to each other.

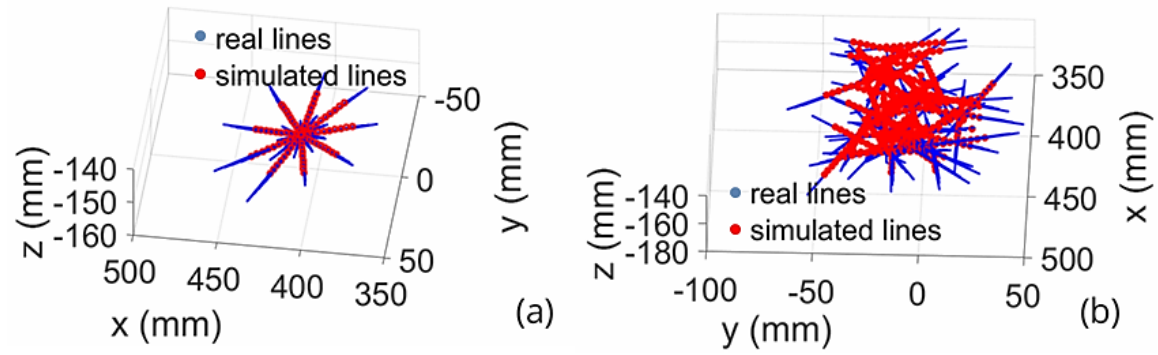

Figure 6. Visualisation of the simulated data and the reconstruction of the real collected data on the same plot (a) circular pattern data (b) random lines on a single plane.

Lastly, the fourth data set was collected from the plane as positioned for the second and third data sets, and based on the judgement of the robot operator 100 random sensor poses were used. In the absence of pre-generated poses by simulations and inspired by the optimisation results, the height, projection and tilt angles were varied for scanning each line by the operator. Similar to the other experiments, the scans were repeated five times and five calibration matrices were computed. At each repetition of the experiment, the same line poses were visited by the sensor.

The four sets of experimental data were then analysed to evaluate the repeatability of each calibration approach and the different collection strategies, as explained in the following three sub-sections. 


\subsection{Accuracy}

To compare the accuracy of the different calibration and data collection strategies, a set of test points were reconstructed in the robot base frame using the calibration matrices found for each of the four calibration strategies. A plane was fitted to the reconstructed test points and the spatial deviations from the fitted plane were computed. In order to have a fair accuracy comparison between these strategies, a common set of 48 test points is used; in this case these points were taken from the first set of calibration data collected using the circular pattern. To reconstruct these points into a common $3 \mathrm{D}$ coordinated system the hand-eye calibrations resulting from each of the four strategies were used. A plane was then fitted to the $3 \mathrm{D}$ reconstructed points in each case and the Euclidean distances of the reconstructed points from their orthogonal projections on the fitted plane were computed, the results are presented in Table 4; as can be seen, apart from the threeplane experiment, all other standard deviations of distances are less than $0.01 \mathrm{~mm}$. The circular pattern obtained the minimum deviations of less than $0.004 \mathrm{~mm}$.

When considering the significantly larger error resulting from the three-plane strategy, it is important to note that this error is most likely not a function of the hand-eye calibration algorithm, but rather that it is due to positional errors of the robotic system. The threeplane strategy is most likely to be affected by robot positioning errors as a larger range of joint motions are needed to reach the three orthogonal planes. While the analysis and characterisation of the sources of errors in the robotic system used for this work is out of the scope of this paper, results obtained by the simulation studies of the three-plane calibration approach showed comparable results to the single-plane strategy (see Figure 5). This supports the conclusion that these larger errors are due to the robot positional data rather than the calibration method.

Table 4. The standard deviation in mm of Euclidean distances of 48 reconstructed points from their corresponding orthogonal projections on a fitted plane, using different strategies hand-eye calibrations.

\begin{tabular}{|l|l|l|l|}
\hline $\begin{array}{l}\text { 3-plane } \\
\text { (user acquisition) }\end{array}$ & $\begin{array}{l}\text { 1-plane (computer } \\
\text { generated random } \\
\text { lines) }\end{array}$ & $\begin{array}{l}\text { 1-plane } \\
\text { (circular } \\
\text { pattern) }\end{array}$ & $\begin{array}{l}\text { 1-plane (user } \\
\text { defined random } \\
\text { lines) }\end{array}$ \\
\hline 0.356 & 0.040 & 0.033 & 0.049 \\
\hline
\end{tabular}

\subsection{Repeatability}

To quantify and compare the repeatability of each calibration approach, calibration data collected for each method was captured multiple times as described above. Multiple hand-eye calibration matrices were then computed using data from each acquisition method. The impact of variation in the calibration matrix was then assessed by projecting a point within the coordinate system of the laser sensor into the coordinate system of the robot end-effector (these coordinate systems are illustrated in Figure 2), and then calculating the standard deviation in the resulting position when each calibration matrix is considered. The point chosen is within the nominal working range of the laser sensor and off-set from the central axis of sensor such that it is $120 \mathrm{~mm}$ along the $\mathrm{z}$ axis of the sensor and $24 \mathrm{~mm}$ along the $\mathrm{x}$ axis of the sensor (the axis directions are illustrated in Figure 2). For the data collection strategies based on a single plane, data was repeatedly collected 
five times. In the case of the three-plane experiments, by combining the data from each of the three planes, over five repeated experiments, a total of 125 calibration matrices were obtained. Due to the variation in sample size Bessel's correction was applied to the estimation of standard deviation for each method. The results can be seen in Table 5, quantified by $\mathrm{x}, \mathrm{y}$ and $\mathrm{z}$ components as well as the scalar magnitude. As can be seen in most cases the repeatability is less than $0.1 \mathrm{~mm}$, and the circular pattern strategy has clearly the most repeatable performance with values that are less than $0.01 \mathrm{~mm}$.

Table 5. The standard deviation of 3D coordinates of a transformed point from the sensor coordinate system into the end-effector coordinate system. Hand-eye calibrations computed from four different calibration strategies were used.

\begin{tabular}{|l|c|c|c|c|}
\hline \multicolumn{1}{|c|}{ Calibration strategy } & $\begin{array}{c}\boldsymbol{x} \\
(\mathbf{m m})\end{array}$ & $\begin{array}{c}\mathbf{y} \\
(\mathbf{m m})\end{array}$ & $\begin{array}{c}\mathbf{z} \\
(\mathbf{m m})\end{array}$ & $\begin{array}{c}\text { Magnitude } \\
(\mathbf{m m})\end{array}$ \\
\hline 3-plane (user acquisition) & 0.020 & 0.015 & 0.096 & 0.099 \\
\hline $\begin{array}{l}\text { 1-plane (computer generated } \\
\text { random lines) }\end{array}$ & 0.037 & 0.042 & 0.004 & 0.056 \\
\hline 1-plane (circular pattern) & 0.002 & 0.008 & 0.000 & 0.008 \\
\hline 1-plane (user defined random lines) & 0.003 & 0.012 & 0.031 & 0.033 \\
\hline
\end{tabular}

\subsection{Automatic calibration strategy for reduced user manual settings}

In the physical calibration experiments that were performed in previous two sections, in two cases, data was acquired automatically. This was done by using the robot tool poses $\mathbf{T}_{\mathbf{R B}}^{\mathbf{E F _ { i }}}$ generated by the simulation program. The poses were defined in the robot coordinate system for scanning the lines based on the associated sensor position parameters $(d, \theta, \beta)$. In addition to the scan parameters, the simulation program requires the orientation of the planar surface artefact to be defined with reasonable certainty. This was achieved using a mounting jig to ensure accurate location of the planar artefact relative to the robot in a known orientation. In practical use, it may well be possible to set-up a similar scenario, so that repeat calibrations can be performed automatically. However, for the case of the initial set-up, in which neither the hand-eye calibration is performed, nor the location of the planar artefact is known, this would not be possible. This would result in the need for full user guidance using the random line method which might require the user to set approximately 100 scans which is significant undertaking in terms of the required user guidance. Therefore, to address this issue the feasibility of conducting a hand-eye calibration, for the purposes of initial setup, with minimal user guidance was investigated.

In order to simplify the acquisition process and minimise the manual setting, a simplified acquisition strategy is proposed. This starts by positioning the calibration target plane within the robot workspace ensuring that the tilt of the plane is such that it is rotationally off-set from the robot's global coordinate system by at least one degree about each axis. Then, only four lines must be collected from the tilted plane. The lines should be collected from the middle part of the plane so that, they can be considered on the edges of the area that scans should be performed. As shown in Figure 8, the initial four corner lines are collected by the user. Using a manually estimated hand-eye 
transformation (that could be based on a crude measurement), the scanned lines are 3D reconstructed. Then a plane is fitted to the reconstructed lines allowing simulation of the circular pattern of lines and the corresponding robot poses $\mathbf{T}_{\mathbf{R B}}^{\mathbf{E \mathbf { F } _ { \mathbf { i } }}}$. The generated robot poses are then used to scan the pattern automatically. Due to the use of the crudely estimated hand-eye calibration, the scanned parameters and line poses do not exactly follow the simulations; however, as shown above, the calibration algorithm tolerates deviations from the target line scan pattern, and optimal sensor position parameters. In this case, while the optimal parameters are not exactly followed, their partial variations are still valid and will preserve the full rank input variation criteria. For example, a simulated tilt angle of $120^{\circ}$ might be $117^{\circ}$ in practice, however this shift will also affect the other tilt angles so that their variation with respect to each other is preserved.

Using the proposed method with the four manually scanned lines, hand-eye calibration was performed. The calibration matrix was used to reconstruct the same circular data set that was used for accuracy tests in the previous experiments. The mean and standard deviation of the Euclidean distances of the 3D reconstructed points to the corresponding fitted plane was $0.085 \mathrm{~mm}$ and $0.066 \mathrm{~mm}$ respectively.

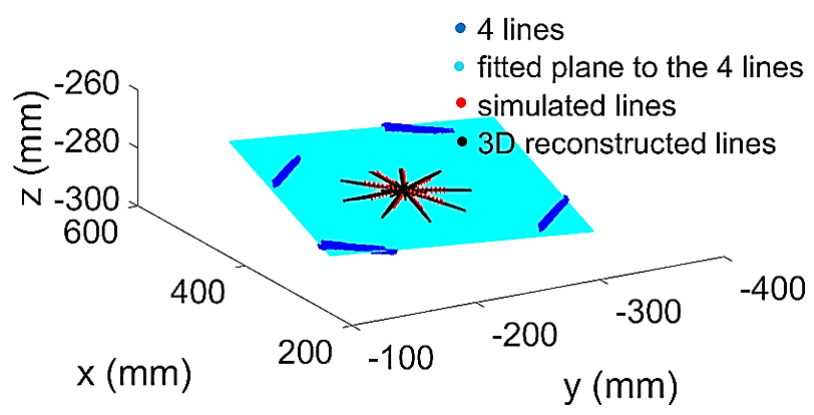

Figure 8. Illustration of the user acquired four lines and the fitted plane. The plane is used to simulate the scan poses for calibration data acquisition automatically. The simulated and reconstructed lines are also shown.

\section{Discussion}

The calibration results of the simulated and real data sets demonstrate the possibility of hand-eye calibration using the single-plane strategy for any laser sensor system. The optimised sensor position parameters for the real sensor system showed promising results in both clean and noisy conditions while using simulated and real data, even while using initial guesses that deviated largely from the true values. As seen in Section 4.1, with the initial guesses defined by adding a uniformly distributed noise that varied by up to ten percent of the true translational and rotational elements, the hand-eye calibration was generally successful for all the 200 different simulated sensor systems.

Through application of the simulation a more optimal set of parameters to define the data collection strategy used in the calibration procedure was defined. While the parameters represent more optimal solutions for the laser sensor and robot used in this work, these were not validated as generally optimal for all robot mounted laser sensors. 
This is because the optimisation was performed for the known system and its related training simulations. Therefore, a general optimum solution in this case must be investigated in future studies.

For the systems simulated in this work, selected ranges of noise included in the simulation experiments also include the same noise level that was used in [27], which was $0.5 \mathrm{~mm}$. While direct comparison with [27] is not possible due to unknown information regarding the exact configuration of the simulation used, the errors using the simulated three-plane data in the current study are in a similar range compared to those in [27] where for a similar noise level of 0.5 an average translation error of around $300 \mu \mathrm{m}-400 \mu \mathrm{m}$ and average angular error of approximately $0.1^{\circ}-0.2^{\circ}$ was shown.

When considering the relative accuracy of the four data collection strategies, the experimental test results clearly show the single-plane methods result in lower residual errors when collected points are compared to a best-fit plane. In the case of the threeplane real experiments, the calibration accuracy seemed to be influenced by the errors in robot positional accuracy, which is thought to be worse as a function of the increased range of robot movements required to perform this calibration. In contrast, as seen in section 5.1, the circular strategy, with the minimum required level of robot movements resulted in lowest reconstruction error. This would suggest there may be an advantage to be gained when doing a hand-eye calibration using a single plane close to the location of subsequent scanning, however this must be investigated more thoroughly in future work.

\section{Conclusion}

In this paper a new strategy for hand-eye calibration of robot mounted laser sensors was proposed. A significant advantage of the new method is that it requires only a single planar artefact, and the user is only required to direct the robot to collect the first four scan lines, with the potential for all subsequent scans to be collected automatically following a fully defined set of test parameters, that can be used to guide the robot automatically. The strategy is based on a previously developed algorithm for hand-eye calibration that requires the use of three planes, however, through the rigorous definition of the calibration data collected, the conditions needed for a robust Least Squares solution using only a single plane have been fully defined in this work.

To aid the collection of good quality calibration data, a new design for the location of target lines that follow a circular pattern was proposed. In addition, a simulation approach was used to select optimal parameters to define the poses of the laser sensor relative to the target plane, resulting in a defined set of 48 sensor poses from which the circular pattern of lines should be scanned. This approach was shown to efficiently allow maximum variation of the calibration data collected from a $2 \mathrm{D}$ plane, and thus it improves the performance of the calibration in terms of both accuracy and repeatability.

The work also concluded that the position of the plane in the global coordinate system of the robot must be carefully considered, and small plane rotation angles of less than one degree about each axis of the global coordinate system should be avoided. These small angles lead to non-convergence of the calibration algorithm, or a converged but erroneous solution; by following the guidelines proposed in the paper these troubling situations can be easily avoided. 
The resulting calibration strategy is simple from the perspective of user interaction, robust, accurate, and requires only a simple single plane artefact; making the approach low cost and easy to implement for many industrial applications of robot deployed laser scanning.

\section{Funding}

The authors acknowledge the Centre for Innovative Manufacturing in Intelligent Automation at Loughborough University for providing access to the robot used in this work, and the funding from the Engineering and Physical Research Council in the UK under grant EP/L01498X/1.

\section{References}

[1] A. Brunete, E. Gambao, J. Koskinen, T. Heikkilä, K.B. Kaldestad, I. Tyapin, G. Hovland, D. Surdilovic, M. Hernando, A. Bottero, S. Anton, Hard material small-batch industrial machining robot, Robot. Comput. Integr. Manuf. 54 (2018) 185-199. doi:10.1016/J.RCIM.2017.11.004.

[2] S. Sharifzadeh, I. Biro, N. Lohse, P. Kinnell, Abnormality detection strategies for surface inspection using robot mounted laser scanners, Mechatronics. 51 (2018) 59-74. doi:10.1016/j.mechatronics.2018.03.001.

[3] P.K. Sara Sharifzadeh, Istvan Biro, Niles Lohse, Robust Surface Abnormality Detection for a Robotic Inspection System, in: IFAC-PapersOnLine, 2016: pp. 301-308.

[4] C. Yu, J. Xi, Simultaneous and on-line calibration of a robot-based inspecting system, Robot. Comput. Integr. Manuf. 49 (2018) 349-360. doi:10.1016/J.RCIM.2017.08.006.

[5] A. Rout, B.B.V.L. Deepak, B.B. Biswal, Advances in weld seam tracking techniques for robotic welding: A review, Robot. Comput. Integr. Manuf. 56 (2019) 12-37. doi:10.1016/J.RCIM.2018.08.003.

[6] X. Chen, A.G. Dharmawan, S. Foong, G.S. Soh, Seam tracking of large pipe structures for an agile robotic welding system mounted on scaffold structures, Robot. Comput. Integr. Manuf. 50 (2018) 242-255. doi:10.1016/J.RCIM.2017.09.018.

[7] W.-L. Li, H. Xie, G. Zhang, S.-J. Yan, Z.-P. Yin, Hand-Eye Calibration in Visually-Guided Robot Grinding, IEEE Trans. Cybern. 46 (2016) 2634-2642. doi:10.1109/TCYB.2015.2483740.

[8] H. Xie, C. Pang, W. Li, Y. Li, Z. Yin, Hand-eye calibration and its accuracy analysis in robotic grinding, in: 2015 IEEE Int. Conf. Autom. Sci. Eng., IEEE, 2015: pp. 862-867. doi:10.1109/CoASE.2015.7294189.

[9] Q. Zhan, X. Wang, Hand-eye calibration and positioning for a robot drilling system, Int. J. Adv. Manuf. Technol. 61 (2012) 691-701. doi:10.1007/s00170-011-3741-4.

[10] Y.C. Shiu, S. Ahmad, Calibration of wrist-mounted robotic sensors by solving homogeneous transform equations of the form AX = XB, IEEE Trans. Robot. Autom. 5 (1989) 16-29. doi:10.1109/70.88014.

[11] R.Y. Tsai, R.K. Lenz, A new technique for fully autonomous and efficient 3D robotics hand-eye calibration, IEEE Trans. Robot. Autom. 5 (1989) 345-358.

[12] R. Horaud, F. Dornaika, Hand-Eye Calibration, Int. J. Rob. Res. 14 (1995) 195-210. doi:10.1177/027836499501400301.

[13] K. Daniilidis, Hand-Eye Calibration Using Dual Quaternions, Int. J. Rob. Res. 18 (1999) 286-298. doi:10.1177/02783649922066213.

[14] J. Li, M. Chen, X. Jin, Y. Chen, Z. Dai, Z. Ou, Q. Tang, Calibration of a multiple axes 3-D laser 
scanning system consisting of robot, portable laser scanner and turntable, Optik (Stuttg). 122 (2011) 324-329. doi:10.1016/J.JJLEO.2010.02.014.

[15] S.Z. Canduo Shen, A Robotic System for Surface Measurement Via 3D Laser Scanner, in: Proc. 2nd Int. Conf. Comput. Appl. Syst. Model., 2012: pp. 1237-1239. doi:10.2991/iccasm.2012.315.

[16] Y. Ren, S. Yin, J. Zhu, Calibration technology in application of robot-laser scanning system, Opt. Eng. 51 (2012) 114204. doi:10.1117/1.OE.51.11.114204.

[17] X. Xu, D. Zhu, H. Zhang, S. Yan, H. Ding, TCP-based calibration in robot-assisted belt grinding of aero-engine blades using scanner measurements, Int. J. Adv. Manuf. Technol. 90 (2017) 635-647. doi:10.1007/s00170-016-9331-8.

[18] Hanqi Zuang, Yiu Cheung Shiu, A noise-tolerant algorithm for robotic hand-eye calibration with or without sensor orientation measurement, IEEE Trans. Syst. Man. Cybern. 23 (1993) 1168-1175. doi:10.1109/21.247898.

[19] S. Yin, Y. Ren, J. Zhu, S. Yang, S. Ye, A vision-based self-calibration method for robotic visual inspection systems., Sensors (Basel). 13 (2013) 16565-16582. doi:10.3390/s131216565.

[20] A.D. Theodor Borangiu, Anamaria Dogar, Calibration of Wrist-Mounted Profile Laser Scanning Probe using a Tool Transformation Approach ( RAAD 2009 ), in: 18th Int. Work. Robot. AlpeAdria-Danube Reg., 2009: pp. 1-5.

[21] Z.G. Zhenqi Zhu, Qing Tang, Jinsong Li, Calibration of laser displacement sensor used by industrial robots, Opt. Eng. 43 (2004) 12. doi:10.1117/1.1631935.

[22] S. Yin, Y. Guo, Y. Ren, J. Zhu, S. Yang, S. Ye, A novel TCF calibration method for robotic visual measurement system, Optik (Stuttg). 125 (2014) 6920-6925. doi:10.1016/J.IJLEO.2014.08.049.

[23] W. Chen, J. Du, W. Xiong, Y. Wang, S. Chia, B. Liu, J. Cheng, Y. Gu, A noise-tolerant algorithm for robot-sensor calibration using a planar disk of arbitrary 3-D orientation, IEEE Trans. Autom. Sci. Eng. PP (2016) 1-14.

[24] J. Santolaria, J.-J. Aguilar, D. Guillomía, C. Cajal, A crenellated-target-based calibration method for laser triangulation sensors integration in articulated measurement arms, Robot. Comput. Integr. Manuf. 27 (2011) 282-291. doi:10.1016/j.rcim.2010.07.008.

[25] M. Wagner, P. Heß, S. Reitelshöfer, J. Franke, Self-calibration method for a robotic based 3D scanning system, IEEE Int. Conf. Emerg. Technol. Fact. Autom. ETFA. 2015-Octob (2015). doi:10.1109/ETFA.2015.7301508.

[26] Guo-Qing Wei, G. Hirzinger, Active self-calibration of hand-mounted laser range finders, IEEE Trans. Robot. Autom. 14 (1998) 493-497. doi:10.1109/70.678458.

[27] F.B. Carlson, R. Johansson, A. Robertsson, Six DOF eye-to-hand calibration from 2D measurements using planar constraints, IEEE Int. Conf. Intell. Robot. Syst. 2015-Decem (2015) 3628-3632. doi:10.1109/IROS.2015.7353884.

[28] T. Hastie, R. Tibshirani, J. Friedman, The Elements of Statistical Learning, Springer New York, New York, NY, 2009. doi:10.1007/978-0-387-84858-7.

[29] D.W. Eggert, A. Lorusso, R.B. Fisher, Estimating 3-D rigid body transformations: a comparison of four major algorithms, Mach. Vis. Appl. 9 (1997) 272-290. doi:10.1007/s001380050048.

[30] J.R. Hodgson, P. Kinnell, L. Justham, N. Lohse, M.R. Jackson, Novel metrics and methodology for the characterisation of 3D imaging systems, Opt. Lasers Eng. 91 (2017) 169-177. doi:10.1016/J.OPTLASENG.2016.11.007.

[31] J.R. Hodgson, P. Kinnell, L. Justham, M.R. Jackson, Characterizing the influence of surface roughness and inclination on 3D vision sensor performance, in: Proc. SPIE - Int. Soc. Opt. Eng., 2015. doi:10.1117/12.2228826.

[32] P. Kinnell, I. Biro, S. Sharifzadeh, Calibration test data for a robot mounted laser scanner, (2018). 
doi:10.17028/rd.lboro.7365020.v1. 